\title{
A Sampling Theorem for the Radon Transform of Finite Complexity Objects
}

\author{
Irena Maravić ${ }^{\dagger}$ Martin Vetterli ${ }^{\dagger}$ \\ $\nmid \mathrm{LCAV}$, Ecole Polytechnique Fédérale de Lausanne, Switzerland \\ $\ddagger$ EECS Dept., University of California, Berkeley, USA \\ \{Irena.Maravic, Martin.Vetterli\}@epfl.ch
}

\begin{abstract}
We present sampling results for certain classes of two-dimensional signals that are not bandlimited, but have a parametric representation with a finite number of degrees of freedom, such as 2-D Diracs, polygons and bilevel signals with piecewise polynomial boundaries. As opposed to standard multidimensional sampling schemes, the proposed methods exploit the properties of the Radon transform of such signals. In particular, we demonstrate that by using an appropriate sampling kernel, one can perfectly reconstruct the signal from a finite set of samples of its Radon transform, and thus significantly reduce a computational load. The novel approach we present in the paper, offers practical algorithmic implementation and is potentially applicable to a large class of two-dimensional signals.
\end{abstract}

\section{Introduction}

Sampling theory has experienced a strong research revival over the past decade, which led to refinement of original Shannon's theory, and development of more advanced formulations with direct impact in signal processing and communications. For example, the traditional sampling paradigm for representation of bandlimited functions can be extended to classes of signals, such as uniform splines, which are not bandlimited but live on a subspace spanned by a generating function and its shifts [3].

Recently, it has been shown that it is possible to develop sampling schemes for a larger class of nonbandlimited signals, such as stream of Diracs, nonuniform splines and piecewise polynomials [4]. A common feature of these signals is that they have a parametric representation with a finite number of degrees of freedom, and can be perfectly reconstructed from a finite set of samples.

On the other hand, while there are many interesting results for one-dimensional signals [4], the problem becomes more involved when going to higher dimensions, and typically does not allow direct extensions of 1-D formulations. Furthermore, most of the multidimensional sampling algorithms encountered in practice still rely on results from a bandlimited case, which may lead to unnecessarily high computational load, particularly for those classes of signals that could presumably be represented by a finite number of samples. Therefore, a very interesting but challenging question is whether it is possible to come up with practical methods for sampling 2-D signals with a finite complexity, that would allow for perfect reconstruction from a finite set of samples. In this paper, we consider the problem of developing sampling schemes and reconstruction formulas for certain classes of such signals, namely 2-D Diracs generated by a Poisson process, polygons and bilevel signals with piecewise polynomial boundaries.

We exploit the properties of the Radon transform of such signals, and demonstrate that by taking a finite number of filtered line integrals, the problem can be reduced to its one-dimensional equivalent, which is much more convenient for algorithmic implementation.

\section{2-D Signals with Finite Complexity}

The class of signals having finite complexity can be defined as the class having a parametric representation with a finite number of degrees of freedom. The main purpose for introducing the complexity (i.e. the rate of innovation) $\rho$, is that it can often be directly related to the minimum sampling rate, or the minimum number of samples that leads to perfect reconstruction. Consider for example a two-dimensional bandlimited signal $g(x, y)$, with the Fourier transform that is nonzero over a finite region $R$ in the frequency space $\left(f_{x}, f_{y}\right)$. If we let $2 B_{x}$ and $2 B_{y}$ represent the widths in the $f_{x}$ and $f_{y}$ directions of the smallest rectangle that encloses the region $R$, then the signal can be perfectly 
represented by properly spaced samples,

$$
g(x, y)=\sum_{m=-\infty}^{\infty} \sum_{n=-\infty}^{\infty} g_{m n} \operatorname{sinc}\left(\frac{x-m X}{X}, \frac{y-n Y}{Y}\right)
$$

where $X$ and $Y$ are such that $X \leq \frac{1}{2 B_{x}}$ and $Y \leq \frac{1}{2 B_{y}}$, and $g_{m n}=g(m X, n Y)$. One possible interpretation of this result is that the bandlimited signal $g(x, y)$ can be considered as having $1 / X$ and $1 / Y$ degrees of freedom per unit of length in the $x$ and $y$ directions respectively. A more general form of the above expression for $g(x, y)$, which extends the subspace of bandlimited functions, is obtained by replacing the function $\operatorname{sinc}(x, y)$ by a more general template, the so-called generating function $\varphi(x, y)$

$$
g(x, y)=\sum_{m=-\infty}^{\infty} \sum_{n=-\infty}^{\infty} c_{m n} \varphi\left(\frac{x-x_{m}}{X}, \frac{y-y_{n}}{Y}\right)
$$

with $x_{m}$ and $y_{n}$ being arbitrary shifts. When $\varphi(x, y)=\delta(x, y)$ and both $x_{n}-x_{n-1}$ and $y_{n}-y_{n-1}$ are i.i.d. random variables with exponential density, then $g(x, y)$ describes the 2-D Poisson process. Examples of other classes include simple lines and polygonal lines, planar parametric curves, as well as bilevel signals whose boundaries have a finite number of degrees of freedom. While developing exact sampling formulas for a very general case may be rather intricate, we believe that the approach we present in the paper can be extended to a large class of signals of finite complexity.

\section{Finite Set of 2-D Diracs}

Consider a signal $g(x, y)$ made up of $M$ Diracs generated by a 2-D Poisson process. Even though this signal has a simple parametric representation, its algebraic structure provides a good insight into the fundamental principles inherent in the algorithms for more complicated signals. The concept we present is based on sampling the Radon transform of the signal $g(x, y)$, and offers a possibility of decomposing the problem into a set of 1-D equivalents, along with all the interesting extensions that would entail.

Let the signal $g(x, y)$ be represented as

$$
g(x, y)=\sum_{k=0}^{M-1} c_{k} \delta\left(x-x_{k}, y-y_{k}\right)
$$

and let $R g(p, \theta)$ be the Radon transform of $g(x, y)$ [1]

$$
R g(p, \theta)=\int g(x, y) \delta(p-x \cos (\theta)-y \sin (\theta)) d x d y
$$

that is, the integral of $g$ over the line $l_{p, \theta}$ defined by $p(\theta)=x \cos (\theta)+y \sin (\theta)$. For any given angle $\theta_{0}$, the Radon transform $R g\left(p, \theta_{0}\right)$ can be represented as a weighted sum of at most $M$ 1-D Diracs, since there can be more than one Dirac spike on the path of integration

$$
R g\left(p, \theta_{0}\right)=\sum_{k=0}^{M_{0}-1} a_{0 k} \delta\left(p-p_{0 k}\right)
$$

where $M_{0} \leq M$.

Since the signal made up of $M 1-\mathrm{D}$ Diracs can be perfectly recovered from a set of $2 M$ samples [4], by using either the sinc or the Gaussian sampling kernel, we can use that result to develop a sampling scheme for 2-D signals as well. Namely, instead of taking the line integral in equation (3), we can replace the $\delta$ function by an appropriate kernel, such as the sinc function. In other words, we can consider the filtered projection $\widetilde{R} g(p, \theta)$ of the signal $g(x, y)$

$$
\widetilde{R} g(p, \theta)=\int g(x, y) B_{p} \operatorname{sinc}\left(B_{p}\left(p-p_{\theta}\right)\right) d x d y
$$

where $p_{\theta}=x \cos (\theta)+y \sin (\theta)$. An interesting property emerging form this formulation is that for any angle $\theta_{0}$, the projection $\widetilde{R} g\left(p, \theta_{0}\right)$ of $g(x, y)$ is a convolution of its Radon transform $R g\left(p, \theta_{0}\right)$, which is a stream of 1-D Diracs, and the sinc sampling kernel, i.e.

$$
\widetilde{R} g\left(p, \theta_{0}\right)=R g\left(p, \theta_{0}\right) * B_{p} \operatorname{sinc}\left(B_{p} p\right)
$$

where $B_{p}$ is a bandwidth of the sinc sampling kernel (to be chosen appropriately). The above equation implies that the locations $p_{k}$ and weights $a_{k}$ of the 1-D Diracs, defined by (4), can be obtained from $N \geq 2 M_{0}$ samples of $\widetilde{R} g\left(p, \theta_{0}\right)$, that is, $p_{n}\left(\theta_{0}\right)=$ $\widetilde{R} g\left(p-n T_{p}, \theta_{0}\right), n=0, \ldots N-1$, where $T_{p}=1 / B_{p}$ [4]. An outline of the main steps of the algorithm is given in the Appendix, while a more detailed discussion on this subject can be found in $[2,4]$.

While the set of locations $\left\{p_{0 k}\right\}$ does not itself define $g(x, y)$, we will prove that the projection of $g(x, y)$ onto $M+1$ lines entirely specifies the signal. Assume therefore that we do the projection of $g(x, y)$ onto $M+1$ lines with different slopes, determined by angles $\theta_{0}, \theta_{1}, \ldots \theta_{M}$. By using the method described above, we can solve for the coordinates $p_{m k}$ and weights $a_{m k}$, $m=0,1, \ldots M$ of the $1-D$ Dirac streams along each line, and thus uniquely specify the set of "projecting" lines $l_{p_{m k}, \theta_{m}}$. Clearly, for any point that belongs to the set of $2-\mathrm{D}$ Diracs, exactly $M+1$ projecting lines must intersect. A reverse statement, that the points 


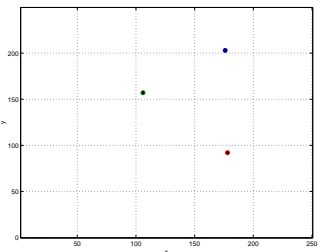

(a)

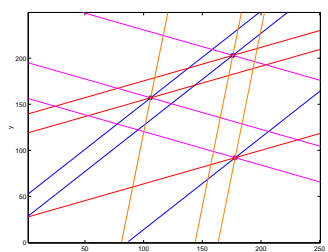

(b)
Figure 1: Finite Set of 2-D Diracs. (a) 2-D signal consisting of $M=3$ weighted Diracs (b) Reconstruction of the signal from projecting lines. Points where exactly $M+1=4$ lines intersect correspond to the Diracs in the set.

where $M+1$ projecting lines intersect must belong to the set, can be proved by counterexample. Namely, assume that exactly $M+1$ such lines intersect at some point $A$. If $A$ doesn't belong to the set of Diracs, then there must be at least one point from the set located at each of the lines $l_{p_{m k}, \theta_{m}}, m=0,1, \ldots M$, which implies that $g(x, y)$ is being made up of at least $M+1$ Diracs, and that obviously contradicts our basic assumption. The weights $c_{k}$ can be found by solving the system of $M$ linear equations that we can choose out of $(M+1) 2 M$ equations (available from the set of projections of $g(x, y))$. Thus we can state:

Theorem Consider a finite set of $M$ weighted 2-D Diracs and let $\varphi(p)$ be the 1-D sinc sampling kernel with bandwidth $B_{p}$. If $N \geq 2 M$ then the $N$ sample values of the filtered Radon transform

$$
<R g\left(p, \theta_{m}\right), B_{p} \operatorname{sinc}\left(B_{p}\left(p-n T_{p}\right)\right)>n=1, \ldots N
$$

taken along each of the $M+1$ different directions $\theta_{0}$, $\theta_{1}, \ldots \theta_{M}$, uniquely specify the signal.

Note that a similar result holds for the Gaussian kernel as well.

A simulation example that illustrates the performance of the proposed sampling scheme is presented in Figure 1. The Radon transform of a signal made up of $M=3$ weighted Diracs is sampled by the Gaussian kernel, with six uniform samples $(N=2 M)$ taken along each of the $M+1=4$ different directions. The signal is perfectly reconstructed from the set of projecting lines, namely, the points where exactly four lines intersect correspond to locations of the Diracs, as shown in Figure 1(b).

The above algorithm yields a unique solution by taking on the order of $M^{2}$ samples of the Radon transform. However, in some cases of more complicated signals the algorithm's complexity remains either the same or can be even reduced, with the only difference being the use of an alternative sampling kernel, as will be shown in the sequel.

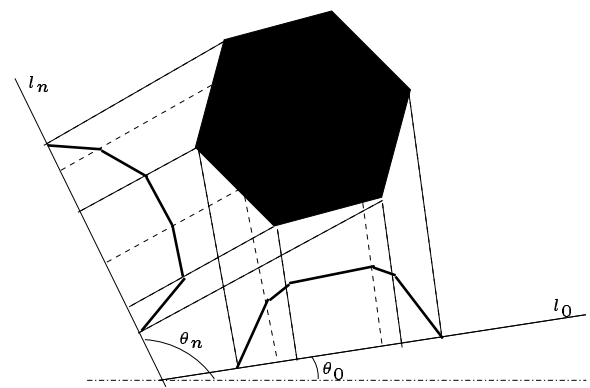

Figure 2: Bilevel Polygon The projection of a bilevel polygon onto an arbitrary line is a piecewise linear signal. For the signal with $M$ vertices, $2 M$ sample values of the filtered Radon transform taken along each of the $M+1$ different directions uniquely specify the signal.

\section{Extension of the results on the 2-D Poisson Process}

After the somewhat "synthetic" case study of the previous section, we will next examine extensions of this result that are of greater practical importance. In the first example we will consider the problem of sampling a bilevel polygon, while the second application is related to bilevel signals with piecewise polynomial boundaries. By exploiting the property that the signals have a finite number of degrees of freedom, we will develop sampling methods that are much more efficient than the existing schemes in terms of computational requirements.

\subsection{Bilevel Polygon}

Consider a signal $g(x, y)$ that is a bilevel polygon, and let vertices be at points $\left(x_{i}, y_{i}\right), i=1,2, \ldots M$. Clearly, the signal is uniquely specified by coordinates of its $M$ vertices, thus it has $2 M$ degrees of freedom. An elegant way to solve for the coordinates $\left(x_{i}, y_{i}\right)$, takes advantage of the fact that the projection of $g(x, y)$ onto an arbitrary line is a piecewise linear signal, as illustrated in Figure 2.

We can therefore incorporate the sampling schemes for such 1-D signals into our algorithm and replace the $\delta$ function from (4) by a proper kernel. Namely, a 1-D piecewise linear signal $f(p)$ having $M$ pieces, can be uniquely represented by its $2 M$ samples $<f(p), \varphi^{(2)}\left(p-n T_{p}\right)>$, where $\varphi^{(2)}(p)$ is the second derivative sinc sampling kernel given by $\operatorname{IFT}\left\{(j \omega)^{2} \operatorname{rect}\left(\frac{\omega}{2 \pi B_{p}}\right)\right\}$, with $B_{p}=\frac{1}{T_{p}}$ [4]. Due to the associativity of the convolution operator, a convolution of the signal $f(p)$ with $\varphi^{(2)}(p)$ is equivalent to the convolution of the second derivative $f^{(2)}(p)$ (i.e. a stream of Diracs) with the sinc kernel, thus the problem can be reduced to the one we analyzed in the previous section. Therefore we have 
Proposition 1 Given a bilevel polygon with $M$ vertices and the second derivative sinc sampling kernel $\varphi^{(2)}(p)$ with $B_{p}=\frac{1}{T_{p}}$, then the $N \geq 2 M$ samples

$$
<R g\left(p, \theta_{m}\right), \varphi^{(2)}\left(p-n T_{p}\right)>n=1, \ldots N
$$

taken along each of the $M+1$ directions $\theta_{0}, \theta_{1}, \ldots \theta_{M}$, are a sufficient representation of the signal.

\subsection{Bilevel signal with Piecewise Polyno- mial Boundary}

Define a bilevel signal with a piecewise polynomial boundary with respect to the $x$ axis as

$$
g(x, y)= \begin{cases}1 & y \leq p(x) \\ 0 & \text { otherwise }\end{cases}
$$

where $p(x)$ is a piecewise polynomial signal having $M$ pieces of maximum degree $R$. We can directly use the result from the 1-D case, and take the samples of the projection of $g(x, y)$ on the $x$-axis, by using the $(R+1)$ th derivative sinc kernel $\varphi^{(R+1)}(x)$. Since the $(R+1)$ th derivative $p^{(R+1)}(x)$ is a collection of at most $M$ weighted Diracs, we need to take only $2 M$ samples in order to recover the signal [4], thus we have

Proposition 2 Consider a bilevel signal with a piecewise polynomial boundary $p(x)$ having $M$ pieces of maximum degree $R$. The set of $N \geq 2 M$ samples,

$$
<R g\left(p, \theta_{x}\right), \varphi^{(R+1)}\left(p-n T_{p}\right)>n=1, \ldots N
$$

taken along the $x$-direction, uniquely represents the signal.

\section{Conclusion}

We have developed algorithms for sampling certain classes of 2-D signals that are not bandlimited, yet have a finite number of degrees of freedom. The proposed sampling schemes exploit that property, and in a noiseless case lead to perfect reconstruction from a finite number of samples of the Radon transform. In order to derive exact sampling formulas, we used some techniques already encountered in the context of spectral estimation. The proposed algorithms are very convenient in terms of computational efficiency, and are potentially of impact in certain signal processing applications, such as reconstruction from projections. Finally, we believe that a large class of sampling problems can be analyzed within the proposed framework, opening up an area for further investigation.

\section{APPENDIX}

The result we used in the proof of Theorem 1 , namely that it is possible to solve for the locations and weights of the 1-D Diracs, defined by (4), from
$N \geq 2 M$ samples of $\widetilde{R} g\left(p, \theta_{0}\right)$, can be derived as follows. Consider the sample values $p_{n}\left(\theta_{0}\right)$

$$
\begin{aligned}
p_{n}\left(\theta_{0}\right) & =\sum_{k=0}^{M_{0}-1} a_{0 k} B_{p} \operatorname{sinc}\left(p_{0 k} / T_{p}-n\right) \\
& =(-1)^{n} \sum_{k=0}^{M_{0}-1} \frac{a_{0 k} B_{p} \sin \left(\pi p_{0 k} / T_{p}\right)}{\left(\pi p_{0 k} / T_{p}-\pi n\right)}
\end{aligned}
$$

If we define a Lagrange polynomial $P_{k}(u)=\frac{L(u)}{u-p_{0 k} / T_{p}}$ where $L(u)=\prod_{k=0}^{M_{0}-1}\left(u-p_{0 k} / T_{p}\right)$, then multiplying both sides of (8) by $P(n)$, we obtain an expression in terms of the interpolation polynomials:

$$
(-1)^{(n+1)} L(n) p_{n}\left(\theta_{0}\right)=\sum_{k=0}^{M_{0}-1} a_{0 k} B_{p} \sin \left(\frac{\pi p_{k}}{T_{p}}\right) \frac{L_{k}(n)}{\pi}
$$

Since the right-hand side of the above equation is a polynomial of degree $M_{0}-1$ in the variable $n$, then applying $M_{0}$ finite difference makes the lefthand side vanish, namely, $\Delta^{M_{0}}\left((-1)^{n} L(n) s_{n}\right)=0$, $n=M_{0}, \ldots N-1$. Therefore, if we let $L(u)=\sum_{k} l_{k} u^{k}$, the following relation must hold

$$
\sum_{k=0}^{M_{0}-1} l_{k} \Delta^{M_{0}}\left((-1)^{n} n^{k} p_{n}\left(\theta_{0}\right)\right)=0
$$

or written in a matrix form,

$$
\mathrm{V} \cdot \mathbf{l}=\mathbf{0}
$$

where $\mathbf{V}$ is an $\left(N-M_{0}\right) \times\left(M_{0}+1\right)$ matrix. The system (10) has a non-trivial solution if $N-M_{0} \geq M_{0}$ and the $\operatorname{rank}(\mathbf{V}) \leq M_{0}$. Equation (10) can therefore be used to solve for the locations $p_{k}$ of the 1-D Diracs. Finally, the corresponding weights $a_{0 k}$ can be found from the system of equations defined in (8), which completes the proof.

\section{References}

[1] S. R. Deans and R. Moses, The Radon Transform and Some of Its Applications, Willey, 1993.

[2] P. Stoica and R. Moses, Introduction to Spectral Analysis, Prentice Hall, 2000.

[3] M. Unser, "Sampling-50 years after Shannon", Proceedings of the IEEE, Vol. 88, No. 4, pp. 569587, April 2000.

[4] M. Vetterli, P. Marziliano and T. Blu, "Sampling signals with finite rate of innovation", IEEE Transactions on Signal Processing, submitted May 2001. 